\title{
Research on the Ethicalized Effect of Martial Arts Elements on the Violence Aesthetics in Chinese Films
}

\author{
Luoxuan $\mathrm{Zhou}^{1}{\text { Baojun } \mathrm{Chu}^{1, *} \text { Suyalema Huo }}^{1}$
}

\author{
${ }^{1}$ Xiamen Academy of Arts and Design, Fuzhou University, Xiamen, Fujian, China \\ *Corresponding author. Email: t17075@fzu.edu.cn
}

\begin{abstract}
Violence aesthetics in films is the integration of violence and film art, and beautification of violence images is an important means of designing films with violence aesthetics. Films with violence aesthetics provide the audience with a way to appreciate the beauty of violence, which is sought after by a large number of audiences in China and abroad. Meanwhile, it also arouses scholars' thinking on the negative effects. That is, as a kind of violent media, audiences' long-term or short-term exposure to a large amount of violent contents in films will increase the probability of committing aggressive behaviors and cause social problems. By analyzing and comparing the compatibility between the characteristics and functions of martial arts elements in Chinese films with violence aesthetics and the information ethicalization theory, this paper utilizes the ethicalized characteristics of martial arts elements to add them to the design of violence scenes in such films. It is hoped that the negative impact of films with violence aesthetics on the audience's psychology can be reduced appropriately, so as to provide reference for the design of violence scenes in these films in the future.
\end{abstract}

Keywords: martial arts elements, violence aesthetics, information ethicalization

\section{INTRODUCTION}

Violence is an instinct stimulated by human beings in primitive society, including killing, fighting and slashing, etc. It is the embodiment of human aggression and attack consciousness. Aggression is a human instinct. Art is a tool for human expression. By using art to express violence and watch violence in art, human beings can release their aggression and meet their psychological needs. Such a background of demand gives birth to films with violence aesthetic as the main artistic expression method. As the audience scope and influence of films with violence aesthetic continue to expand, the negative impact of violence audiovisual materials on the audience has aroused extensive discussions among scholars from all fields. Martial arts originated from violence, but after thousands of years of development andits integration with traditional Chinese culture, it no longer belongs to the category of pure violence, but turns to the direction of art. Whether martial arts, as an artistic manifestation of violence, can improve the negative impact of violence aesthetics films on the audience is a worthy direction to be studied.

\section{FILM VIOLENCE AESTHETICS AND CHINESE MARTIAL ARTS}

\section{A. The violence aesthetics in films}

Violence aesthetics is the integration of violence elements and film art, which really entered the public's vision in the 1980s. It originated in the United States and later developed and matured in Hong Kong, China [4]. Violence aesthetics does not belong to the strict aesthetic theory system. It is defined as a formalized representation of violent scenes in films. The beautification presentation of violent elements such as blood, fight and mutual injury is an indispensable part of films with violence aesthetics. However, the presentation form of these elements is usually exaggerated and against the common sense, highlighting the formalistic beauty of violence, and avoiding the moral thinking behind violence.

As an important region for the development and growth of violence aesthetics, China and the United States show two different forms of violence beauty due to different cultural backgrounds and ideas:

The violent images in American films with violence aesthetic are often presented in the form of jokes, emphasizing contrast and irony. In films, violence symbols and comedy symbols are usually combined to show a formal beauty with a strong sense of conflict. 
Taking, for example, a scene in Quentin Tarantino's "Pulp Fiction" in which two characters discuss the details of a robbery in a restaurant, the relaxed and comfortable visual sense of the clip is in great contrast to the tense and exciting robbery content in the dialogue, so that when the two suddenly act violently after the conversation, there is an extreme beauty of contrast and conflict.

The Chinese films with violence aesthetics adopt the dance forms featuring the beauty of movement, modeling and artistic conception in the expression of violence pictures. It emphasizes the philosophical implication under the representation of violence, uses the classical aesthetic feeling in the traditional martial arts moves and movements, and integrates the Chinese national spirit into the design of fighting movements to show the artistic conception beauty of fighting and fighting behaviors. Taking Zhang Yimou's Film "Shadow" as an example, in the action design of the decisive battle between Jingzhou and General Yang, Jingzhou uses umbrella as a weapon, making the action design very flexible with strong feminine characteristics; General Yang wears a long knife and his body is covered with armor, whose movements are designed to highlight the sense of weight and strength. The design of the fight movement of the two men contains a large number of tai Chi elements of "Yin and Yang opposition, conquering the unyielding with the yielding", showing a strong Taoist ideological characteristics, emphasizing the beauty of artistic conception.

\section{B. The characteristics of traditional Chinese martial arts}

The origin of martial arts is violence. It originates from the action of fighting and attacking between people in primitive society. It is the law of attacking and defending summed up in attack and combat. However, martial arts are not the same as the violent incident of mutual attack. In essence they are a kind of martial art combining the historical fighting routine and the traditional Chinese philosophy.

1) Martial arts have the characteristics of art, and the form of their movements is the fighting rules bordering on dance: It is a summary of the law of attack and defense in combat, with corresponding forms of attack and defense. Different regions and schools have their own distinctive martial arts routines with different elements as the core, representing a different approach to offense and defense. With the civilization of the society and the restriction of social legal system and morality on people, martial arts, after thousands of years of development and precipitation, is no longer a tool for people to fight against the enemy. It is transformed into an ornamental performance activity instead. Through the evolution and arrangement of the movement, the aesthetic feeling of the movement is expressed. The design of the movement emphasizes the narrative function of the movement symbol, and gradually integrates into the category of art. Its function is weakened, and more emphasis is placed on the artistry of the movement.

2) Martial arts has cultural connotation and is the concrete manifestation of traditional Chinese philosophy: Martial arts movements not only represent the law of attack and defense, and striking action, but also have profound cultural connotation. After absorbing a large number of Taoist theories, martial arts gradually formed an internal and external self-refining mode integrating morality cultivation and temperament cultivation of self, emphasizing the Taoist philosophy of "unity of nature and man", "coping with all motions by remaining motionless", "conquering the unyielding with the yielding", and "gaining mastery by striking only after the enemy has struck". [6] The moves and routines of martial arts follow the thinking mode of Taoism. For example, the moves of Tai Chi emphasize the need for remaining motionless and waiting for motions of the enemy, and most of its moves consist of attacking first and defending later [7].

\section{Chinese martial arts films with violence aesthetic}

Martial arts have unique advantages in the combination with violence aesthetics and film in those they are formalized and artistic violence. The movements and actions of modern martial arts have been beautified and improved, and integrated into the ornamental value of dance, which has a high aesthetic value. As a kind of visual symbol integrated by various elements of Chinese culture, martial arts is highly ornamental.

Taking martial arts as a form of violence, it can create martial arts films with violence aesthetics that are more suitable for Chinese film and television environment.

Chinese martial arts films with violence aesthetics have unique action design characteristics. The combat action design in such films is reflected in the combination of philosophy, traditional culture, dance movements and attack and defense routines. In the process of martial arts, movements emphasize the beauty of dynamic lines, shapes and artistic conception. Each movement has a strong philosophical implication and classical cultural flavor. It is an integration of art and skill, and has a strong aesthetic feeling of form.

Chinese martial arts films have unique character design characteristics. The element of "xia (a kind of chivalrous swordsman in ancient China)" is one of the main elements of martial arts films with violence 
aesthetic. The heroic image, personality spirit and ideology of "xia" contain some cultural norms and national spirit of the Chinese nation [5]. The element of "xia" is a feature that distinguishes Chinese martial arts films with violent aesthetic from western films with violent aesthetic. "Xia" represents the heroic image of "loyalty and courage, righteous spirit and sacrifice one's own interests for the sake of others". It weakens the immorality of films with violent aesthetic and adds ethical concepts to the audience's acquisition of violent content, which is a kind of resolution to violence.

\section{MARTIAL ARTS ELEMENTS AND INFORMATION ETHICALIZATION IN FILMS WITH VIOLENCE AESTHETICS}

\section{A. Psychological problems caused by films with violence aesthetics}

According to the existing studies on violence in media, watching a lot of violent content in media will increase the probability of audience's aggressive behaviors [15]. With the development of information technology, film and other new media industries are constantly making progress, and the sense of immersion and reality brought to the audience is also constantly strengthened. While such progress meets the needs of some audiences to watch violent content, a series of problems also appear — some of the information presented in the films with violence aesthetics does not have ethical concepts and moral constraints. When the media follow the audience's preferences without thinking and massively produce such content with violence aesthetics, a negative impact will be brought on the audience's psychology, which will even lead to social violence: according to the cultivation theory of communication, when audiences are exposed to violent audiovisual environment for a long time, they will show more violent tendency. Therefore, long-term viewing of films with violent content will have a cultivation effect on the audience, and there is a certain probability that it will lead to aggressive behaviors of the audience, giving rise to the occurrence of social violence [15]. However, short-term viewing of films with violent content also has a negative effect on the audience's psychology. In his General Aggression Model, Anderson mentioned that the short-term impact of violent media on the audience will lead to an increase in aggressive emotions [10] and the increase of hostile cognitive bias, and previous studies have shown that hostile cognitive bias is significantly correlated with aggressive behavior and can positively predict the aggressive behavior of audiences [11]. Therefore, when the audience's hostile cognitive bias increases, it will also increase the probability of audience's aggressive behavior.

\section{B. Information ethicalization and martial arts elements in Chinese films with violence aesthetics}

Florridi puts forward the methodology of information ethics in his book Information Ethics: "Moral behavior itself can now be modeled as an information process, that is, a series of messages (M), called by A (information carrier), leading to the transformation and impact on the state of $\mathrm{P}$ (information transmission)... Messages with multiple transformations may respond to $\mathrm{M}$, depending on how P's methods interpret M." [2]

This methodology expounds the possible effect of moral behavior on information communication form after participating in media information communication - it is a philosophical theory that specially explores information technology and the social ethical problems it causes [12], and resolves the social problems brought by media in the form of information ethics. With the emergence of new media, considering the impact of its powerful expression on the audience, information needs to be involved in a certain constraint mechanism in the process of dissemination. It can be a powerful weapon to reduce the negative effects of violent content in films by using moral and ethical concepts to involve in the dissemination of violent information in films with violent aesthetic.

The characteristics of martial arts elements in Chinese violence aesthetics movies share a similar vein with the idea of information ethicalization: film is an information medium with information technology characteristics; violence aesthetics is a technique of expression that emphasizes the formal beauty of film violence, blood and fighting scenes, and contains a large number of violent elements; so long-term viewing of films with violent content by the audience will produce cultivation effects on the audience, and there is a certain probability that they will appear aggressive behaviors, leading to the occurrence of social violence [15]; however, short-term viewing of films with violent content also has a negative effect on the audience's psychology, affecting the audience's aggressive emotion and hostile cognition, and bringing about social ethical problems.

Originated in violence, martial arts are different from violence and they are formalized and artistic violence. With the civilization of society and the restriction of social legal system and morality on people, martial arts, after thousands of years of development and accumulation, is no longer a tool for people to fight against the enemy and become a performance activity with ornamental value. Through the evolution and arrangement of the movement, the aesthetic feeling of the movement is expressed. The design of the movement emphasizes the narrative function of the movement symbol, and gradually integrates into the category of art. Its functionality is 
weakened, and more emphasis is placed on the artistry of the movement. Martial arts also incorporated a lot of spiritual connotation and philosophical implication of "pursuing goodness and accumulating virtue" in traditional Chinese culture, which weakened the immorality of violent aesthetic films. Adding ethical ideas to the violent content shown to the audience beautifies the presentation form of inappropriate elements in the violent picture and is a kind of digestion of violence. By presenting the violent elements in films with violence aesthetic in the form of martial arts, it has the characteristics of information ethics, which is conducive to reducing the negative influence brought by films with violence aesthetic and strengthening the adaptability of films with violence aesthetic under the Chinese film and television system.

Therefore, in the design of violence aesthetics films, the ethical effect of martial arts may be used to reduce the negative impact of violence aesthetics films on the audience's psychology, compared with the use of western boxing, Sanda and other violent elements.

\section{EXPERIMENT ON THE ETHICALIZATION EFFECT OF MARTIAL ARTS ELEMENTS}

\section{A. Experimental subjects and experimental design}

A total of 111 WeChat users over 18 years of age from all over the country who have movie-watching experience and volunteer to participate in the experiment were selected as preliminary experimental subjects through the Internet. The preparatory experimental subjects were divided into two experimental control groups, A and B. 48 preparatory experimental subjects in Group A were required to fill in the Chinese version of Buss \& Perry aggression questionnaire A; 63 test subjects in Group B were asked to complete the Chinese version of Buss \& Perry aggression questionnaire $B$. According to the questionnaire results, the groups $\mathrm{A}$ and $\mathrm{B}$ were excluded from the top $25 \%$ of the total score and the bottom $25 \%$ of the population with low and high aggression tendency, so as to exclude the influence of the difference of aggression tendency on the experiment. In addition, 8 invalid questionnaires were excluded. Finally, 48 people were selected as the official experimental subjects, and the follow-up experiments were conducted in groups A and B. Group A is martial arts violence group, with a total of 24 people, including 12 male and 12 female; Group B is a non-martial arts violence group, with a total of 24 people, including 14 males and 10 females. At the end of the experiment, the experimenter will give the subjects some reward to express their gratitude.

\section{B. The questionnaire}

1) Chinese version of Buss \& Perry aggression questionnair $A / B$ : The questionnaire is from the "Chinese Version of Buss Perry Aggression Questionnaire" revised by Li Xianyun et al, and is used to measure the aggressive tendency of the experimental subjects. There were 29 items in the questionnaire, with 5 options ranging from 1 to 5 . The experimental subjects chose their degree of conformity with the options according to their own conditions, with 1 indicating complete non-conformity and 5 indicating complete conformity. The score of each subscale together and the total score of the scale are the sum of the scores of the items contained therein. The higher an individual's score, the more aggressive he or she is [13].

2) Hostile Cognitive Bias Measurement Questionnaire $A / B$ : After the subjects watched the experimental materials in the groups, they were asked to fill in the "Hostile Cognitive Bias Measurement Questionnaire", which consisted of one reading material and seven selected items. The reading material comes from "Xiao Li's Story" adapted by Cheng $\mathrm{Su}$ from Southwest University. After reading the material, the subjects rated seven choices - empathetic, unfriendly, hostile, friendly, distasteful, considerate, and angry on an 11-point scale, indicating how well they thought Xiao Li matched on the adjectives. 0 means "totally inconsistent" and 10 means "perfectly consistent". Since the level of hostile cognition reflects the probability of the audience's aggressive behavior to a certain extent, this scale is used to record the hostile cognitive bias of the two groups of subjects after viewing the experimental materials, so as to measure the ethicalization effect of martial arts elements.

\section{Experimental material}

1) Experimental materials of group A: Group A is martial arts violence group. The experimental materials are 10 minutes of fighting from Zhang Yimou's film "Shadow". The segment has obvious characteristics of martial arts and acrobatic fighting. The action shows the beauty of poetry and contains a large number of scenes of blood, fighting and death, which conforms to the summary of the characteristics of martial arts violence aesthetic films in this paper. Therefore, this section is selected as the experimental material of Group A.

2) Experimental materials of group B: Group B is the non-martial arts violence group. The experimental materials are selected from the 10-minute fighting fragments in Quentin Tarantino's "Kill Bill", which included a large number of scenes of blood, fighting and death, as well as non-martial arts violent elements 
such as boxing and Sanda. This film has been mentioned as a representative work of violence aesthetics for many times in 248 papers of Core Chinese journals in the paper library of Wanfang, Weipu and Zhiwang, etc., among which the violent fragments are representative to a certain extent. Therefore, this film is selected as the experimental material of Group B.

\section{Implementation of the experiment}

The experimental subjects all adopted the method of individual experiment. From the results of the Chinese Buss \& Perry attack questionnaire A and B, 24 official subjects were selected from each group A and B. Subjects in group A watch the experimental materials of Group A respectively. After watching the materials, they fill in the hostile cognitive bias measurement questionnaire A within 1-2 minutes. After completing the questionnaire, the subjects in group A receive a certain reward; subjects in group B watched the experimental materials of group B respectively. After watching the materials, they fill in the hostile cognition deviation measurement questionnaire $B$ in 1-2 minutes. After completing the questionnaire, the subjects in group B receive a certain reward. After the end of the experiment, the filling time of questionnaire $\mathrm{A}$ and $\mathrm{B}$ for the measurement of hostile cognitive bias is analyzed, and 4 invalid questionnaires are excluded from Group A, 4 from Group B, and thus 20 valid questionnaires from group A and Group B respectively.

\section{E. Analysis of experimental results}

The mean score (M) and mean difference (SD) of each option in the questionnaire for the measurement of hostile cognitive bias in group A and Group B are shown in "Table I". As can be seen from the M and SD values of the questionnaire results, the mean total score of group A is lower than that of group B, and the mean value of group A is lower than that of group B in the four options of hostile, disgusting, considerate and thoughtful.

\begin{tabular}{|c|c|c|c|c|}
\hline & \multicolumn{2}{|c|}{$\begin{array}{l}\text { Group A } \\
n=20\end{array}$} & \multicolumn{2}{|c|}{$\begin{array}{c}\text { Group B } \\
n=20\end{array}$} \\
\hline & $M$ & SD & $M$ & SD \\
\hline Angry & 5.65 & 2.77 & 5.60 & 2.69 \\
\hline Hostile & 4.95 & 2.33 & 5.05 & 2.76 \\
\hline Unfriendly & 6.75 & 2.36 & 5.65 & 2.86 \\
\hline Disgusting & 6.10 & 2.40 & 6.55 & 2.65 \\
\hline Friendly & 2.60 & 2.15 & 2.70 & 2.12 \\
\hline Considerate & 2.35 & 2.17 & 1.70 & 1.73 \\
\hline Thoughtful & 2.55 & 2.29 & 2.35 & 3.00 \\
\hline Total & 45.95 & 11.27 & 46 & 11.44 \\
\hline
\end{tabular}

It can be seen that compared with non-martial arts films with violence aesthetics, Chinese martial arts films with violence aesthetics can bring less hostile cognitive bias to the audience, which has a certain ethicalization effect.

But the experiment also has some limitations. For example: first, the experiment takes the form of online one-to-one experiment, which to some extent reduces the interference caused by multi-person watching on the experimental subjects, but the interference caused by the surrounding environment of the experimental subjects cannot be excluded; second, although both scales were applicable to all groups of people, the field, education level and experience of the subjects could influence their judgments; third, since the experimental film materials are not specially shot by the author, but are taken from existing resources, which cannot exclude the influence of director level and style on the experiment; fourth, the experimental number is small, resulting in no $\mathrm{p}$ value difference in experimental data.

\section{CONCLUSION}

Through the focus on the formalistic beauty of violence content, films with violence aesthetics use contrast, irony and art to present the conflict and poetic beauty of violence, the terrifying element. While bringing visual and psychological stimulation to the 
audience, they enable the audience to express their violent emotions in a reasonable way and satisfy their curiosity about violence. This novel experience has made violent aesthetic films popular with a large number of moviegoers in China and abroad. But the repeated presentation of too much violence will bring a series of negative effects to the audience. Long-term exposure to violent media will have a cultivation effect, making the audience behave aggressively in real life and causing social problems; short-term exposure to violent media will also enhance the audience's aggressive emotion and hostile cognition, affect the audience's integration into the society, and also increase the probability of audience's aggressive behaviors.

Information ethics is a philosophical theory that specifically explores information technology and the social ethical problems it causes [12]. According to the theory of information ethicalization, the engagement of the concepts of morality and ethics in the dissemination of violence content in violence aesthetic films in the form of information can be an important theoretical basis for reducing the social problems caused by violence in films.

Although martial arts are born from violence, they are not equivalent to violence. They are poetic and artistic violence and have high aesthetic value. They contain a lot of traditional Chinese spiritual connotations and are the figurative mimicry of traditional Chinese culture. Both the philosophical implication between the forms of martial arts moves and the spiritual connotation behind its "xia" elements coincide with the information ethicalization theory of information ethics. Through experiments, it is proved that compared with western films with violence aesthetics that present violent elements in the forms of boxing, Sanda and so on, martial arts are used to present violent elements in Chinese violence aesthetics films, and its violent elements bring less hostile cognitive bias to the audience. Therefore, compared with the presentation forms of violent elements such as western boxing and swordsman, martial arts elements have ethical effects, which can reduce the negative impact of violent aesthetic films on the audience.

\section{References}

[1] Konrad Lorenz. On Aggression [M]. Trans. Wang Shouzhen, Wu Yuejiao. Beijing: The Writers Publishing House, 1987: 271

[2] Lucinano Floridi. The Ethics of Information [M]. Hoven M.J.van den, John Weckert. Information Technology and Moral Philosophy [M]. Trans. Zhao Yinghuan, Song Jixin, Zhang Qin. Beijing: Science Press, 2014: 43-46.

[3] Kang Er. Another Quest into "What Is Art?" [J]. Jiangsu Provincial Academy of Social Sciences, 2012(04): 189-196. (in Chinese)
[4] Hao Jian. The Sense of Form, Psychological Mechanism and Social Cognition of "Violence Aesthetics" [J]. Journal of Beijing Film Academy, 2005, (no. 4). (in Chinese)

[5] Sun Gang. Philosophical Reflection on Martial Arts Film and Television Images and Martial Arts [J]. Film Literature, 2010(08): 16-17. (in Chinese)

[6] Xing Qing. Influence of Taoist Thought on Chinese Martial Arts Culture [J]. Forum on Chinese Culture, 2014(12): 159-161. (in Chinese)

[7] Tian Jinlong. Study on the Principles of Pair Excersise of the Rank Taijiquan Sets [J]. Journal of Xi'an Institute of Physica Education, 2014, 31(03): 334-337+349. (in Chinese)

[8] Tang Zhiyun, Zhou Bin. Violence Aesthetics in Chinese Martial Arts Films [J]. Contemporary Literary Criticism, 2014(04): 104 107. (in Chinese)

[9] Zhong Zhijin. Effects of Violent Games on Players' Violent Tendencies: Theory, Method and Conclusion [J]. Journalism Bimonthly, 2010(04): 94-98+118. (in Chinese)

[10] Guo Xiaoli, Jiang Guangrong, Zhu Xu. Acta psychologica sinica,2009,41(03):259-266. Short-term desensitization effects of violent video games: a comparison of two contact modes [J] Acta Psychologica Sinica, 2009, 41(03): 259-266. (in Chinese)

[11] Li Zhuoyang, Zhang Ye. Relationship Between Hostile Cognitive Bias and Aggressive Behavior in College Students [J]. Shanxi Youth, 2018, (the 5th issue). (in Chinese)

[12] Wu Yidi. Moral Dilemma and Transcendence of Violent Computer Games — Based on Miguel Sicart's Model and Methods [J]. Journal of Northeastern University (Socia Science), 2019, (No. 5). (in Chinese)

[13] Li Xianyun, Fei Lipeng, Zhang Yali, Niu Yajuan, Tong Yongsheng, Yang Shaojie. Revision and Reliability and Validity of the Chinese Version of Buss and Perry Attack Questionnaire [J]. Chinese Journal of Nervous and Mental Diseases, 2011, (10). (in Chinese)

[14] Cheng Su. Effects of Self-affirmation on Subjective and Behavioral Responses to Social Exclusion [D]. Chongqing: Southwest University, 2011. DOI:10.7666/ D.Y1882680. (in Chinese)

[15] Anderson CA, Bushman BJ. Effects of Violent Video Games on Aggressive Behavior, Aggressive Cognition,Aggressive Affect,Phesiological Arousal, and Prosocial behavior: A Metaanalytic Review of the Scientific Literature[J]. Review of the Science, 2001, 12(5): 353-359. 